\title{
Fiber Laser Based Hydrophone Systems
}

\author{
Asrul Izam AZMI ${ }^{1}$, Ian $\mathrm{LEUNG}^{1}$, Xiaobao $\mathrm{CHEN}^{2}$, Shaoling ZHOU ${ }^{2}$, \\ Qing $\mathrm{ZHU}^{2}$, Kan $\mathrm{GAO}^{2}$, Paul $\mathrm{CHILDS}^{3}$, and Gangding PENG ${ }^{1}$ \\ ${ }^{1}$ School of Electrical Engineering and Telecommunications, The University of New South Wales, NSW, 2052, Australia \\ ${ }^{2} 23 r d$ Research Institute, China Electronic Technology Group Corporation, Shanghai, 200437, China \\ ${ }^{3}$ Department of Electronics, Tsinghua University, Beijing, 100084, China \\ *Corresponding author: Gangding PENGＥ-mail: g.peng@unsw.edu.au
}

\begin{abstract}
We report our recent work on distributed feedback fiber laser based hydrophones. Some issues related to sensitivity, such as fiber laser phase condition, demodulation, and packaging, are also discussed. With the development of appropriate digital signal processing (DSP) techniques and packaging designs, an interferometric-type distributed feedback (DFB) fiber laser hydrophone system with acoustic sensitivity of $58.0 \mathrm{~dB} \cdot \mathrm{re} \cdot \mu \mathrm{Pa} \cdot \mathrm{Hz}^{-0.5}$ at $1 \mathrm{kHz}$ or a minimum detectable acoustic pressure below $800 \mu \mathrm{Pa}$ during field test is attained. We have also investigated an intensity-type DFB fiber laser hydrophone system and its performance.
\end{abstract}

Keywords: Fiber laser, hydrophone, interferometric, acoustic sensitivity

\section{Introduction}

Many applications are now employing optical fiber sensors in preference to conventional electronic sensor. This tendency has been driven by unsubtle advantages of optical fiber, which is frequently quoted as immunity to electromagnetic interference and disturbance, compact in-fiber design, and relative simplicity for multiplexing. One of the areas that have been continuously attracted interest over the last three decades is optical fiber hydrophone. Currently, optical fiber hydrophone has been evolved into some of the most demanding applications, ranging from military sonar $[1,2]$ to geophysical [3] applications. Also strong interest has been focused on new photonic sensors incorporating digital techniques that can further reduce cost and improve reliability of data acquisition operations compared to traditional analog circuit.
For deep sea applications, the sensitivity of hydrophone system should be the same as the background acoustic noise level of quiet ocean, represented by deep sea state zero (DSS0) to be $100 \mu \mathrm{Pa}$ at $1 \mathrm{kHz}$. Hence the development of fiber optic hydrophone systems concerns with high sensitivity, operational depth, dynamic range, applicable frequency range, and operating costs. Practical large multi-channel systems deploy hydrophones in array, and have further considerations such as the ease of repairing, simplicity of manufacturing, ruggedness to survive in hostile environments, and robustness for handling during deployment and recovery.

In recent years, digital signal processing (DSP) has been applied for post-detection processing [4-6]. One main advantage of using DSP is that the performance and functions of the systems can be modified only by software changes, which is much easier, quicker and more inexpensive than replacing

Received: 1 December 2010 / Revised version: 9 January 2011

(C) The Author(s) 2011.This article is published with open access at Springerlink.com 
components in analog circuits. Packaging technique is essential in many aspects [7-10], where it can greatly improve sensitivity, flatten frequency response, and provide protection to bare fiber to survive in harsh underwater environment. The most recent packaging designs such as air backed plastic mandrel and flexural beam based air filled housing improve sensitivity of bare fiber up to $30 \mathrm{~dB}[7,8]$ and $70 \mathrm{~dB}$ respectively [9]. Recently, we have also developed a new scheme using composite cavity fiber laser to provide additional means of sensitivity improvement [11]. Integration of these two components (i.e. DSP and packaging) is therefore essential in realizing high performance fiber laser hydrophone system.

This paper is organized as follows: in Section 2, the approaches used in analyzing fiber laser phase and gain conditions are presented. The response of fiber laser implies the sensitivity expectation of interferometric based sensing system. In Section 3, the interferometric based sensing system together with digital demodulation technique is discussed. Experimental results obtained from field test are also presented in Section 3. Finally in Section 4, we present an intensity-type based hydrophone system and the related experimental results.

\section{Fiber laser phase and gain conditions}

Different approaches in analyzing wavelength and intensity responses for different fiber lasers are described. The distinct approaches are due to different assumptions made of field propagation in the structure. For distributed feedback fiber laser (DFBFL), electric field is considered spatially distributed within gratings, making analysis more complex using coupled mode theory (CMT). However for distributed Bragg reflector fiber laser (DBRFL) and composite cavity fiber laser (CCFL), simple approximation can be made based on multiple reflection phenomena, leading to simpler analytical model. Distinguished intensity and wavelength responses can be observed between single cavity and composite cavity laser. In this section, we describe the model of phase and conditions of different laser structures used for analyzing wavelength and intensity responses.

\subsection{Distributed feedback fiber laser}

As illustrated in Fig. 1, a DFBFL is formed by a $\pi$-phase shifted fiber Bragg grating (FBG) written on a continuous length erbium doped fiber (EDF). It is very stable single longitudinal mode source, with narrow linewidth (between $1 \mathrm{kHz}$ and $10 \mathrm{kHz}$ ) [12, 13], and low phase noise (about $0.3 \mu \mathrm{rad} \cdot \mathrm{Hz}^{-0.5} \cdot \mathrm{m}^{-1}$ ) [14]. A stable single longitudinal mode is accomplished from the design of laser with a very short cavity (of $\pi$-phase shift). The comparison of field mode distribution of DFBFL and DBRFL obtained from numerical calculation is also shown in Fig. 1. Such concentrated mode around $\pi$-phase shift makes DFBFL less affected by external perturbations such as temperature gradient and physical actions (bending or twisting), but in return it has lower slope efficiency, compared to DBRFL. Typically, DFBFL has length of several centimeters up to $10 \mathrm{~cm}$ [15]. Longer cavity has advantages in term of achieving narrower linewidth [16], and provides better stability against external reflection [17]. However from the sensing perspective, it is more advantageous to have shorter cavity to keep the sensor head as compact as possible and reduce pump absorption which is critical for arrayed system.

There are a few cavity designs for different applications that have been demonstrated over the past years: 1) apodization for suppression of higher order lasing mode [18, 19] and optimizing output power [20], with previous work obtained about 50-dB suppression using Gaussian apodization, 2) dual phase-shifted distributed feedback (DFB) to create stable dual wavelength operation [21, 22], and 3) smoothed phase shift transition to improve resistance to cross-coupling that increases phase noise [23]. 


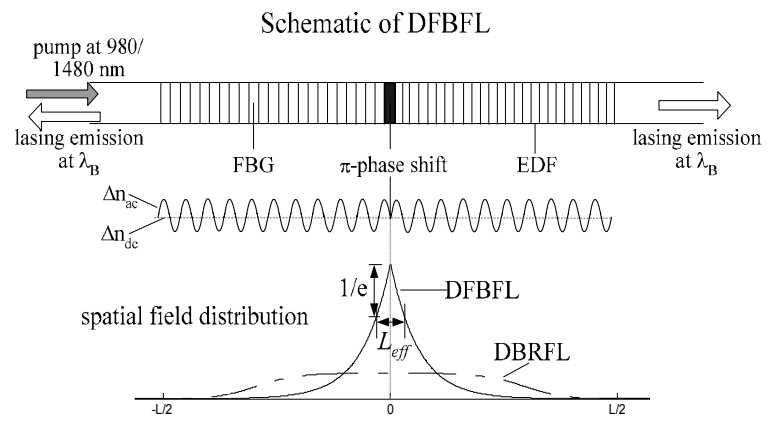

(a)

Wavelength $(\mathrm{nm})$

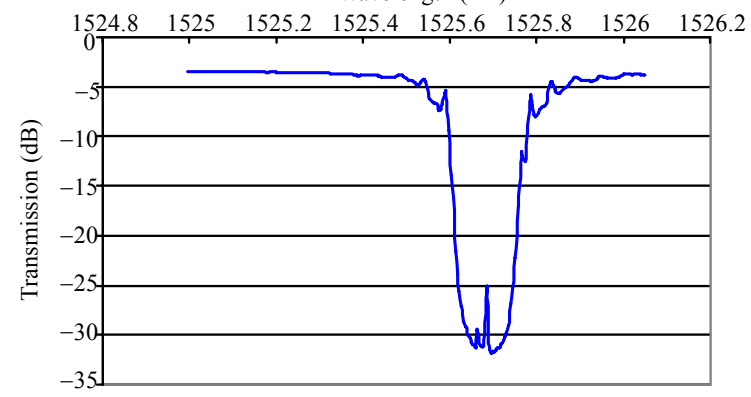

(b)

Fig. 1 (a) Schematic diagram of a DFBFL and (b) transmission spectrum of a fabricated 3-cm DFBFL.

Coupled mode theory (CMT) [24] is the most common method in analyzing spatially distributed field of periodic structure such as DFBFL. This treatment is different from CCFL and DBRFL since we assume that the field is spatially distributed within the gratings for DFBFL. For uniform structure of DFBFL, the phase and gain condition expression derived from CMT [25] can be written as

$$
\left[\frac{S-(\gamma-i \Delta \beta)}{S+(\gamma-i \Delta \beta)}\right] e^{2 S L}=-1 .
$$

In (1), $S$ is given by

$$
S=\sqrt{\kappa^{2}-(\Delta \beta+i \gamma)^{2}}
$$

and $\Delta \beta$ is the detuning of propagation constant from the designed value given by

$$
\Delta \beta=2 \pi n_{\mathrm{eff}}\left(\frac{1}{\lambda_{L}}-\frac{1}{\lambda_{B}}\right) .
$$

The remaining symbols are defined as follows: $\gamma$ is linear gain, $\kappa$ is coupling coefficient, $n_{\text {eff }}$ is effective refractive index, $\lambda_{B}$ is designed Bragg's wavelength, $L$ is total cavity length, and $\lambda_{L}$ is lasing wavelength. Lasing wavelength and threshold gain of DFBFL are obtained by solving (1).
Although CMT provides good insights into laser physical properties, the analysis will be limited only for uniform structure. For spatially non-uniform structures, e.g. DFBFL with apodization, chirped grating, or multiple phase-shifted grating, the numerical method becomes an essential tool. In this case, transfer matrix method (TMM) [19] derived from CMT is required for analysis.

\subsection{Distributed Bragg reflector and composite cavity fiber laser}

Cavity of DBRFL is simply formed by two FBGs inscribed on a continuous EDF. DBRFL is also a single cavity laser which has similar type as DFBFL, but it has significantly longer effective cavity length. This results in a more efficient pump-lasing power conversion. Previously, incorporation of an external reflector to distributed Bragg reflector (DBR) lasers has been studied for several purposes including linewidth narrowing [26, 27], reduction of low frequency wavelength instability [28], and continuous wavelength tuning $[29,30]$. It will be demonstrated by inclusion of an active feedback (hence composite cavity) that phase condition of laser can be significantly altered which is useful for wavelength-type sensing. CCFL schematic and transmission spectrum of a fabricated CCFL are illustrated in Fig. 2.

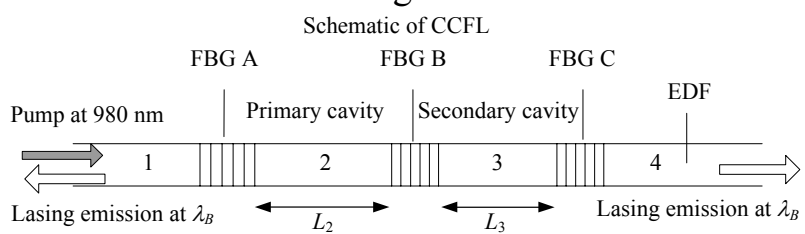

(a)

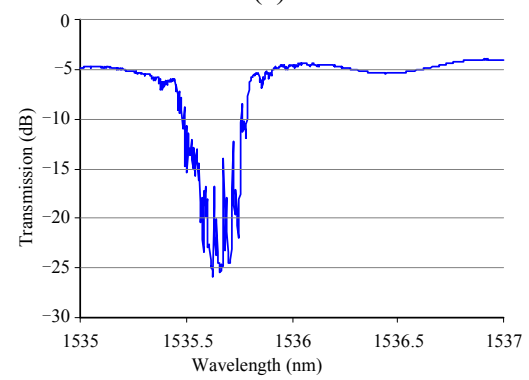

(b)

Fig. 2 (a) Schematic of a CCFL and (b) transmission spectrum of a fabricated CCFL with dimension $L_{2}=2 \mathrm{~cm}$, $L_{3}=8 \mathrm{~cm}$ and all FBGs are $6 \mathrm{~mm}$ in length. 
Following the multiple reflection approach given in $[31,32]$, the boundary value problem of the field is solved, resulting in general phase and gain condition of CCFL given by

$$
r_{A} r_{B} e^{i \phi_{2}} g_{2}-r_{B} r_{C} e^{i \phi_{3}} g_{3}+r_{A} r_{C}\left(2 r_{B}^{2}-1\right) e^{i\left(\phi_{2}+\phi_{3}\right)} g_{2} g_{3}=1
$$

where $\phi_{j}=2 k_{j} L_{j}$ and $g_{j}=\exp \left[2\left(\gamma_{j}-\alpha_{j}\right) L_{j}\right] \quad(j=2$ and 3 denote the respective regions) are round trip phase and round trip gain, $k_{j}$ is propagation constant given by $k=2 \pi n_{\text {eff }} / \lambda_{L}, \gamma_{j}$ is linear gain, $\alpha_{j}$ is linear loss, and $r_{A}, r_{B}, r_{C}$ are amplitude reflectivity of the respective FBGs. By rearranging (4) into real term and imaginary term, the gain and phase for CCFL can be written as

$$
\begin{gathered}
2 \gamma_{\text {th }} L_{2}=2 \alpha_{2} L_{2}-\ln \left(r_{A} r_{B}\right)+G_{C} \\
\phi_{C}=\phi_{2}+p 2 \pi, \quad p: \text { integer } .
\end{gathered}
$$

It is apparent that the first three terms of (5) represent the threshold equation of DBRFL, with $\gamma_{\text {th }}$ denoting the threshold gain. The final term $G_{C}$ is an extra term which is accounted for the effect of active feedback and it is given by

$$
G_{C}=\ln \left|\frac{1-r_{B} r_{C} e^{i \phi_{3}} g_{3}}{1+\frac{r_{C} g_{3}}{r_{B}}\left(1-2 r_{B}{ }^{2}\right) e^{i \phi_{3}}}\right| .
$$

The phase condition in (5) has multiple solutions due to the definition of $p$ as an integer. The lasing mode is selected at the wavelength with the lowest threshold gain.

\subsection{Comparison of wavelengths response to pressure for different fiber lasers}

Wavelength response of single cavity fiber lasers is similar to the FBG, and it is given by [33]

$$
\frac{\Delta \lambda}{\lambda \Delta P}=\frac{(2 v-1)}{E}-\frac{n_{\text {eff }}^{2}}{2 E}(2 v-1)\left(2 \rho_{12}+\rho_{11}\right)
$$

where $P$ is the pressure, $v$ is Poisson's ratio, $E$ is Young's modulus, and, $\rho_{11}$ and $\rho_{12}$ are elasto-optic coefficients. However for composite cavity laser, the response can be drastically changed depending on pressure difference imposed on two cavities. For example, if only one cavity is made responsive and the other cavity is mechanically isolated (hence partial cavity), the response will be significantly different from typical response given in (8). Analysis of pressure responses of different fiber lasers has been carried out by solving the phase and gain conditions of respective lasers. The resulting response of partial cavity sensing of CCFL compared to DFBFL and DBRFL is shown in Fig. 3. For CCFL, physical parameter setup is critical in determining optimum response. The CCFL partial cavity scheme is analyzed with the following parameter values: $r_{B}=0.7, r_{C} g_{3}=0.8, n=1.465, L_{2}=$ $3 \mathrm{~cm}, L_{3}=6 \mathrm{~cm}, v=0.23, \rho_{11}=0.121, \rho_{12}=0.265$, and $E=72 \mathrm{GPa}$. It is apparent that in Fig. 3, the response of partial cavity sensing alternately changes between high and low. The highest responsivity is achieved by primary partial cavity sensing (only $L_{2}$ part is responsive), with the corresponding region marked. The spectral range for high responsivity region is approximate $0.01 \mathrm{~nm}$ (corresponding to about 1-MPa pressure range), providing very large dynamic range of more than $120 \mathrm{~dB}$ as well as good linearity. The highest sensitivity point can be obtained by the adjustment of $r_{C} g_{3}$ parameter (reflectivity of FBG C and gain of region 3 ).

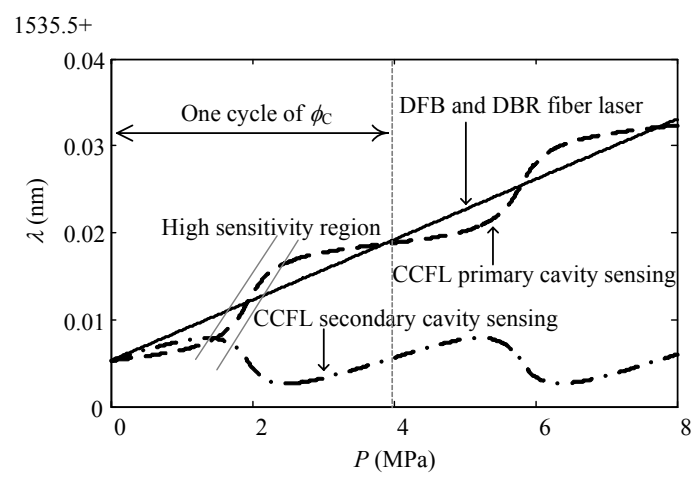

Fig. 3 Comparison of wavelength responses to pressure for different fiber lasers when 8-MPa pressure is scanned through.

\section{Interferometric-type fiber laser hydrophone}

Interferometric optical fiber hydrophone is recognized for achieving the highest sensitivity compared to other types such as intensity [34, 35] and polarimetric hydrophones [36]. The first interferometric-type hydrophone was introduced in 
1977, demonstrated in a bulk optics setup [37]. Since the interferometer output is nonlinear (in cosine term), different demodulation methods have been introduced to extract the output linearly. Commonly known demodulation techniques include: active homodyne with phase tracker [38], passive homodyne [39-41], pseudo heterodyne [42], and synthetic heterodyne [43]. Passive homodyne is normally preferred compared to others as it requires no feedback loop and no reference source for interrogation.

The most successful technique for passive homodyne is based on phase modulation of carrier signal, known as phase generated carrier (PGC). In a pioneering work [39], the demodulation is accomplished by computation on quadrature pair from Bessel's expansion of phase modulated signal. Novel approaches in restraining PGC signal instability caused by changing parameters such as path delay [44], direct current (DC) bias and environmental disturbance [45] have been experimented and proposed recently. The time domain orthogonal sampling approach commercially developed by Optiphase Inc. [40] demonstrates a significant leap of demodulation performance from previous methods with main merits of: very high dynamic range (from micro radians to thousands of radians using fringe counting) and minimizing self crosstalk or noise (since without using harmonics pair). Other method of passive demodulation technique is to use symmetrical $3 \times 3$ coupler $[46,47]$ (i.e. $120^{\circ}$ phase difference of each port), with certain trigonometry operations to linearly resolve phase information.

\subsection{Phase generated carrier demodulation}

In this section, we describe a digital demodulation technique of passive homodyne PGC which is implemented in our hydrophone system. The developed interferometric system which is used to test hydrophone performance is indicated in Fig. 4. In the system, a pump power at $980 \mathrm{~nm}$ is delivered to fiber laser via a wavelength division multiplexing (WDM) coupler and a length of down-lead fiber. Lasing emissions are produced in forward and backward directions and encode the spectral changes due to the acoustic pressure. The interferometer converts the wavelength shift $\Delta \lambda$ into a phase shift $\Delta \phi$ which can be expressed as [48]

$$
\frac{\Delta \phi}{\Delta \lambda}=\frac{2 \pi n_{\mathrm{eff}} d}{\lambda^{2}}
$$

where $d$ denotes the length of delay line. From (8) and (9) the interferometric system phase sensitivity is given by

$$
\frac{\Delta \phi}{\Delta P}=-217+20 \lg (d) \mathrm{dB} \cdot \mathrm{re} \cdot \mathrm{rad} \cdot \mu \mathrm{Pa}^{-1} .
$$

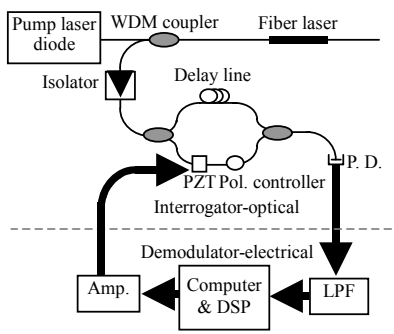

Fig. 4 An interferometric-type fiber laser hydrophone system.

If the system sensitivity is required to match DSS0, the minimum detectable pressure is $100 \mu \mathrm{Pa}$ at $1 \mathrm{kHz}$ and the system will need to be able to detect phase shifts in a noise floor of $-177+20 \lg (d) \mathrm{dB} \cdot \mathrm{re} \cdot \mathrm{rad} \cdot \mathrm{Hz}^{-0.5}$. Such noise figure suggests that sensor enhancements are required to increase the pressure response, ensuring the fiber laser hydrophone suitable for deep sea applications.

A piezoelectric transducer (PZT) phase modulator is inserted into one arm of Mach-Zehnder interferometer (MZI), so that the optical signal phase in that arm can be modulated at carrier frequency $f_{C}$, and the sensing information $\phi(t)$ is carried as the sidebands of the even and odd harmonics of carrier signals. According to Nyquist theorem, $f_{C}$ needs to be at least double the maximum frequency of acoustic signal $f_{A}$ encoded in $\phi(t)$.

In our system, the modulating signal $\cos \left(2 \pi f_{C} t\right)$ is firstly generated digitally using 
Labview and then realized to a voltage waveform by National Instruments PCI 6014 card's on-board digital-to-analog converter. The modulating signal is then amplified in order to drive PZT. The amplifier also performs low pass filter (LPF) on the signal to reduce finite step noise caused by digital-to-analog conversion process.

The variation of the light intensity detected by photodetector can be written as

$$
I_{\mathrm{DET}}(t)=A+B \cos \left[C \cos \left(2 \pi f_{C} t\right)+\phi(t)\right]
$$

where $A$ and $B$ are related to the optical power and mixing efficiency of two arms, and $C$ is the amplitude of sinusoidal signal modulated onto the optical signal. Prior to digitizing, a LPF is required in order to prevent alias signals. According to Nyquist theorem, the relationship between cutoff frequency $f_{\mathrm{CO}}$ and sampling frequency $f_{S}$ is $f_{\mathrm{CO}} \leq f_{S} / 2$. Next, the detector signal is digitized by the same PCI 6014 card, so that $I_{\mathrm{DET}}(t)$ becomes

$$
I_{\mathrm{DET}}[n]=A+B \cos \left(C \cos \left(2 \pi n \frac{f_{C}}{f_{S}}\right)+\phi[n]\right) .
$$

Due to the digitizing process, $I_{\mathrm{DET}}$ is now expressed as a function of data number $n=t f_{S}$, which is dimensionless. As both the fundamental and first harmonic carrier signals are required for demodulation process and according to Nyquist theorem, the sampling frequency must be $f_{S} \geq 4 f_{C}$.
Thus the sampling frequency must be at least eight times the maximum acoustic frequency. The digital computation applied to the digitized signal is shown in Fig. 5. Firstly, two signals of frequencies $f_{C}$ and $2 f_{C}$ are required, which are of the following forms:

$$
\begin{aligned}
& S_{1}[n]=D \cos \left(2 \pi n \frac{f_{C}}{f_{S}}+\theta_{1}\right) \\
& S_{2}[n]=E \cos \left(4 \pi n \frac{f_{C}}{f_{S}}+\theta_{2}\right)
\end{aligned}
$$

where $D$ and $E$ are the amplitudes of $S_{1}[n]$ and $S_{2}[n]$ respectively, and $\theta_{1}$ and $\theta_{2}$ are introduced as the phase differences between $S_{1}$ and $S_{2}$ respectively against the modulating signal.

To reduce complicated synchronization issues, two mixing signals are derived by sampling the modulating signal on a separate input channel of the PCI card. Since $S_{1}[n]$ has the same frequency as the modulating carrier signal, it is readily obtained. $S_{2}[n]$ is then be obtained through trigonometric identity “ $\cos (2 x)=2 \cos ^{2}(x)-1$ ”. First squaring $S_{1}[n]$ to obtain

$$
\left(S_{1}[n]\right)^{2}=\frac{D^{2}}{2}\left(1+\cos \left(4 \pi n \frac{f_{C}}{f_{S}}+2 \theta_{1}\right)\right)
$$

and then removing DC term will result in $S_{2}[n]$ with $E=D^{2} / 2$ and $\theta_{2}=2 \theta_{1}$. Secondly, two signals $S_{1}[n]$ and $S_{2}[n]$ are mixed with $I_{\mathrm{DET}}[n]$. In order to make it easier to comprehend the process, (12) is expanded using Bessel functions to become

$$
\begin{aligned}
& I_{\mathrm{DET}}(t)=A+B\left\{\left[J_{0}(C)+2 \sum_{k=1}^{\infty}(-1)^{k} J_{2 k}(C) \cos \left(4 k \pi f_{C} t\right)\right] \cos (\phi(t))\right. \\
& \left.-\left[2 \sum_{k=0}^{\infty}(-1)^{k} J_{2 k+1}(C) \cos \left((2 k+1) 2 \pi f_{C} t\right)\right] \sin (\phi(t))\right\}
\end{aligned}
$$

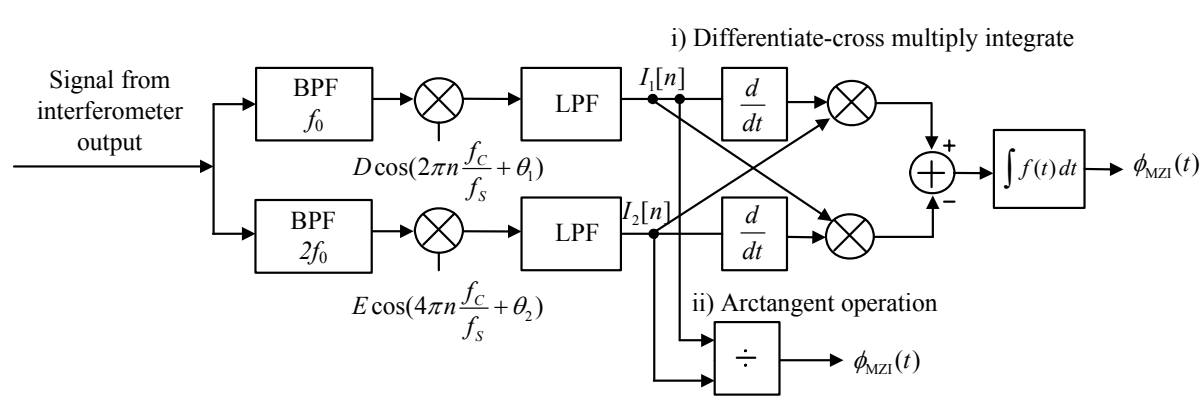

Fig. 5 Diagram of phase generated carrier demodulator implemented in Labview program using i) differentiate-cross multiply integrate and ii) arctangent operation. 
It can be determined that only the components associated with $J_{1}(C)$ and $J_{2}(C)$ in (16) will produce a signal in the base band when being mixed with $S_{1}[n]$ and $S_{2}[n]$ respectively. For the intended purpose of this demodulation process, $I_{\mathrm{DET}}[n]$ for two mixing signals may be simplified to be

$$
\begin{gathered}
I_{D \mathrm{ET} 1}[n]=-2 B J_{1}(C) \cos \left(2 \pi n \frac{f_{C}}{f_{S}}\right) \sin (\phi(t)) \\
I_{\mathrm{DET} 2}[n]=-2 B J_{2}(C) \cos \left(4 \pi n \frac{f_{C}}{f_{S}}\right) \cos (\phi(t))
\end{gathered}
$$

and the signals after mixing can be written as

$$
\begin{aligned}
I_{\mathrm{DET} 1}[n] S_{1}[n] \approx & -B D J_{1}(C) \sin (\phi[n]) \times \\
& \left(\cos \left(4 \pi n \frac{f_{C}}{f_{S}}+\theta_{1}\right)+\cos \left(\theta_{1}\right)\right) \\
I_{\mathrm{DET} 2}[n] S_{2}[n] \approx & -B E J_{2}(C) \cos (\phi[n]) \times \\
& \left(\cos \left(8 \pi n \frac{f_{C}}{f_{S}}+\theta_{2}\right)+\cos \left(\theta_{2}\right)\right)
\end{aligned}
$$

and finally low pass digital filter to extract the base band will recover two orthogonal signals:

$$
\begin{aligned}
& I_{1}[n]=-B D J_{1}(C) \sin (\phi[n]) \cos \left(\theta_{1}\right) \\
& I_{2}[n]=-B E J_{2}(C) \cos (\phi[n]) \cos \left(\theta_{2}\right) .
\end{aligned}
$$

By careful buffering, $S_{1}[n]$ and $S_{2}[n]$ can be delayed independently to make $\theta_{1}$ and $\theta_{2}$ to be close to integer multiple of $\pi$, so that the cosine of these terms can be maximized. The buffering process will also remove the effects of other phase shifts introduced throughout the demodulating process which are not accounted for the derivations.

Two orthogonal signals $I_{1}[n]$ and $I_{2}[n]$ can be applied in two methods to extract the sensing information $\phi[n]$. The first is the cross-multiplying option as shown in Fig. 5. The time derivatives of (21) and (22) are

$$
\begin{gathered}
\frac{\partial I_{1}[n]}{\partial t}=-B D J_{1}(C) \cos \left(\theta_{1}\right) \frac{\partial \phi[n]}{\partial t} \cos (\phi[n]) \\
\frac{\partial I_{2}[n]}{\partial t}=B E J_{2}(C) \cos \left(\theta_{2}\right) \frac{\partial \phi[n]}{\partial t} \sin (\phi[n]) .
\end{gathered}
$$

Cross multiplying $I_{1}[n]$ and $I_{2}[n]$ with their time derivatives gives

$$
\begin{aligned}
I_{1}[n] \frac{\partial I_{2}[n]}{\partial t}= & -B^{2} D E J_{1}(C) J_{2}(C) \times \\
& \cos \left(\theta_{1}\right) \cos \left(\theta_{2}\right) \frac{\partial \phi[n]}{\partial t} \sin ^{2}(\phi[n]) \\
I_{2}[n] \frac{\partial I_{1}[n]}{\partial t}= & B^{2} D E J_{1}(C) J_{2}(C) \times \\
& \cos \left(\theta_{1}\right) \cos \left(\theta_{2}\right) \frac{\partial \phi[n]}{\partial t} \cos ^{2}(\phi[n]) .
\end{aligned}
$$

Then subtracting (25) from (26) gives

$$
\begin{aligned}
& I_{2}[n] \frac{\partial I_{1}[n]}{\partial t}-I_{1}[n] \frac{\partial I_{2}[n]}{\partial t}= \\
& B^{2} D E J_{1}(C) J_{2}(C) \cos \left(\theta_{1}\right) \cos \left(\theta_{2}\right) \frac{\partial \phi[n]}{\partial t} .
\end{aligned}
$$

And finally, integrating (27) gives

$$
I_{\text {OUT }}[n]=B^{2} D E J_{1}(C) J_{2}(C) \cos \left(\theta_{1}\right) \cos \left(\theta_{2}\right) \phi[n]
$$

which is the desired signal $\phi[n]$ multiplied by a constant. For this cross-multiplying option, the integration process will suppress high frequency noise but emphasize low frequency noise.

Further, due to differentiation and integration processes, no absolute DC level can be determined for $\phi[n]$ and signal to noise ratio (SNR) will be decreased for lower frequencies. Slow variations in $B$ due to drift in the interferometer's mixing efficiency can be measured and corrected, but fast variations will add extra noise to the signal due to its squaring effect in (28). If $\theta_{1}$ and $\theta_{2}$ of the multiplying signals are optimized as previously suggested, $\cos \left(\theta_{1}\right) \cos \left(\theta_{2}\right)$ should be close to unity. The product of Bessel functions $\left(J_{1}(C), J_{2}(C)\right)$ can be maximized by choosing $C \approx 2.375$ to be approximate 0.22 .

The second option that can be applied to orthogonal signals to extract $\phi[n]$ is arctangent method. Dividing (21) by (22) gives

$$
\frac{I_{1}[n]}{I_{2}[n]}=\frac{D}{E} \frac{J_{1}(C)}{J_{2}(C)} \frac{\cos \left(\theta_{1}\right)}{\cos \left(\theta_{2}\right)} \tan (\phi[n])
$$

which can be rearranged to recover $\phi[n]$ according to

$$
\phi[n]=\tan ^{-1}\left(\frac{I_{1}[n]}{I_{2}[n]} \frac{E}{D} \frac{J_{2}(C)}{J_{1}(C)} \frac{\cos \left(\theta_{2}\right)}{\cos \left(\theta_{1}\right)}\right) .
$$

If the amplitudes $D$ and $E$ are equal and phases 
$\theta_{1}$ and $\theta_{2}$ are properly optimized and $C \approx 2.63$, so that $J_{1}(C) \approx J_{2}(C) \approx 0.46$, (24) will be simplified to

$$
\phi_{\mathrm{MZI}}(t)=\tan ^{-1}\left(\frac{I_{1}(t)}{I_{2}(t)}\right)
$$

and the display of $I_{1}[n]$ and $I_{2}[n]$ on an $x-y$ graph will show a circle (or part of a circle depending on the amplitude of $\phi[n])$. For this arctangent option, variations of $B$ will not affect the result, and the absolute value of $\phi[n]$ can be obtained. However, care must be taken with the amplitudes $D$ and $E$, phases $\theta_{1}$ and $\theta_{2}$, and choice of $C$ in order to avoid distortions. After $\phi[n]$ is recovered, Fourier transform is performed to time signal to calculate spectral components. The resolution of calculated spectrum is dependent on the sampling frequency and buffer size used to sample the output from photodetector.

Through simulation, it was found that large amplitude sinusoidal and square wave $\phi[n]$ inputs showed the arctangent option to be better at tracking correct amplitude. Also, simulations with Gaussian white noise on the input signal indicated the arctangent option to have lower noise at low frequency and higher noise at high frequency when being compared with the cross-multiplying option. Since neither option can perform better than the other throughout the frequency range of interest, both options were digitally implemented.

\subsection{Field test results}

Extensive laboratory experiments and field tests in lakes have been successfully carried out. A PGC demodulator (explained in Section 3.1) with path imbalance of $150 \mathrm{~m}$ was utilized to examine the hydrophone performance. The benchmark performance is the sensitivity of bare fiber which gives pressure sensitivity of $-168.2 \mathrm{~dB} \cdot \mathrm{re} \cdot \mathrm{rad} \cdot \mu \mathrm{Pa}^{-1}$ at $1 \mathrm{kHz}$. With special DFBFL hydrophone packaging designs, we are able to vastly improve the pressure sensitivity of a bare fiber as well as flattening the frequency response. As indicated in Fig. 6, the average pressure sensitivity from two different packaging designs are -117 and -122 $\mathrm{dB} \cdot \mathrm{re} \cdot \mathrm{rad} \cdot \mu \mathrm{Pa}^{-1}$ respectively, implying enhancement of more than $40 \mathrm{~dB}$ compared to bare fiber.

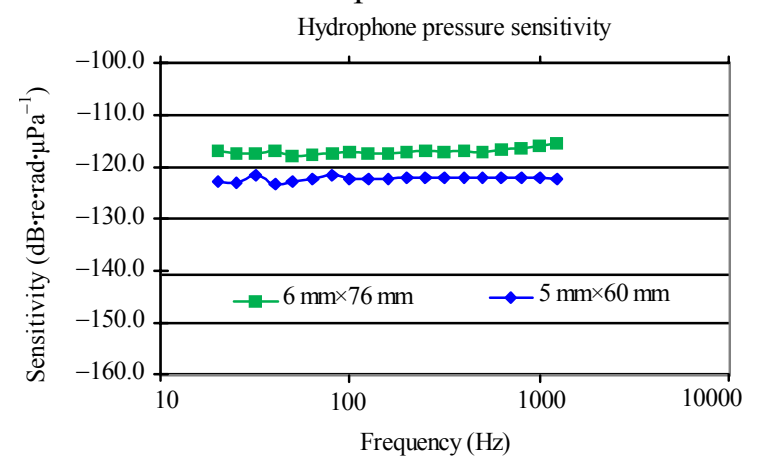

Fig. 6 Pressure sensitivity results of fiber laser hydrophones with two different packaging designs.

Taking into account average pressure sensitivity and on-field phase noise level, noise equivalent pressure (NEP) of hydrophone systems has been determined. The NEP resulted from 4 tests at $1 \mathrm{kHz}$ are $57.8,58.8,57.8$, and $57.5 \mathrm{~dB} \cdot \mathrm{re} \cdot \mu \mathrm{Pa} \cdot \mathrm{Hz}^{-0.5}$, respectively. This produces a very low averaged $\mathrm{NEP}$ at $58.0 \mathrm{~dB} \cdot \mathrm{re} \cdot \mu \mathrm{Pa} \cdot \mathrm{Hz}^{-0.5}$, implying the minimum detectable acoustic pressure of $794 \mu \mathrm{Pa} \cdot \mathrm{Hz}^{-0.5}$. The NEP of hydrophone system obtained from one of the measurement is shown in Fig. 7. Since environment noises during field test are much higher than DSS0, actual minimum detectable pressure should be lower than what we have measured. The ideal NEP supposedly can be determined from anechoic chamber measurement.

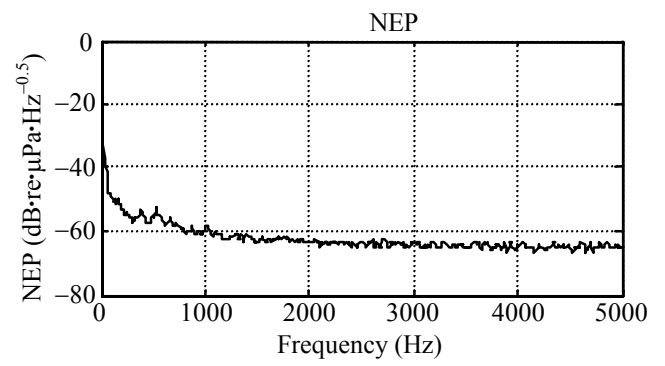

Fig. 7 NEP of the system.

Figure 8 shows the achievement of packaging designs for the last 35 years [49]. Recent published works also have been added to the original figure for comparison. The major players are dominated by defense agencies such as Naval Research Laboratory (USA), Defense Evaluation and Research Agency 
(UK), and lately, Defense Science and Technology Organization (Australia). The increasing sensitivity trend is obvious since early 1990 s, and now the typical sensitivity enhancement is about $40 \mathrm{~dB}$ (100 times) of bare fiber.

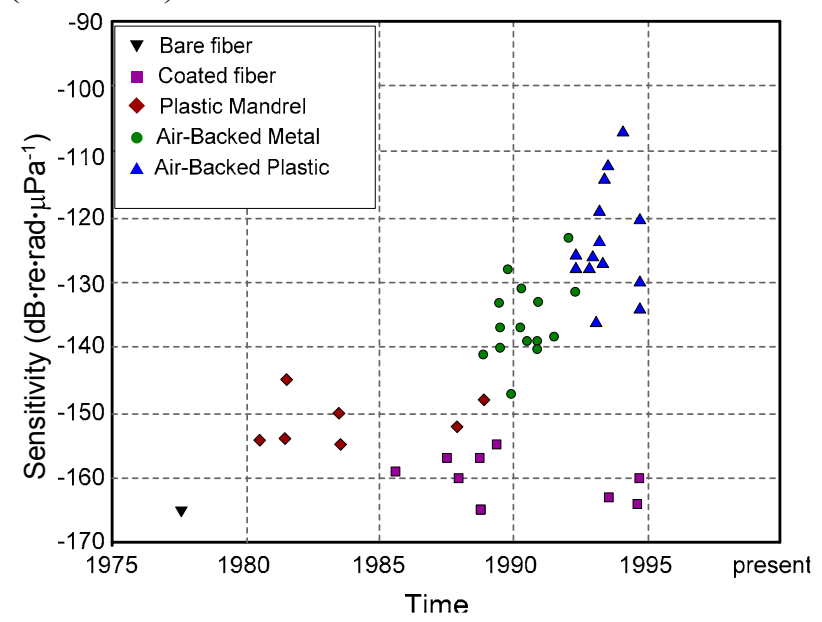

Fig. 8 Progress of interferometric hydrophone system performance in the last 35 years [49].

\section{Intensity-type fiber laser hydrophone}

Fiber lasers can also be used to make intensity-type fiber hydrophones. An intensity-based fiber optic sensing system might be preferred to phase modulated systems in many industrial applications. Due to its active resonance structure and low relative intensity noise, an active fiber laser sensor should be more sensitive and efficient than passive intensity-type sensors. As the pump source and fiber laser technology mature, associated costs should continue to decrease.

An intensity-type fiber laser hydrophone system is shown in Fig. 9. Without MZI section, demodulation becomes more direct and the system is much simpler. Here the photodetector directly converts optical output from fiber laser to electrical signal, which is then digitized after low pass filter to prevent aliasing. The digitized signal is then processed using Fourier transform to evaluate spectral components. During experiment, it was found that an acoustic field modulated the output intensity of a fiber laser, and the spectral information of the acoustic field could also be transformed to calculate spectral components. An acoustic field could be obtained directly from Fourier transform of the intensity with good sensitivity.

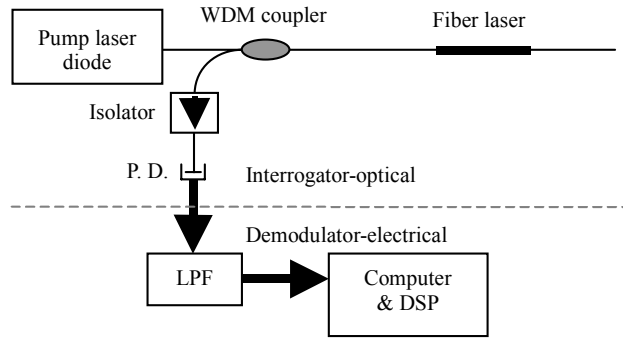

Fig. 9 Setup of intensity modulated fiber laser hydrophone system.

For this system, the sampling frequency needs to be at least double that of maximum acoustic frequency of interest, which is a quarter of the sampling frequency required by an interferometric system. This factor gives a big advantage from reduced hardware requirements.

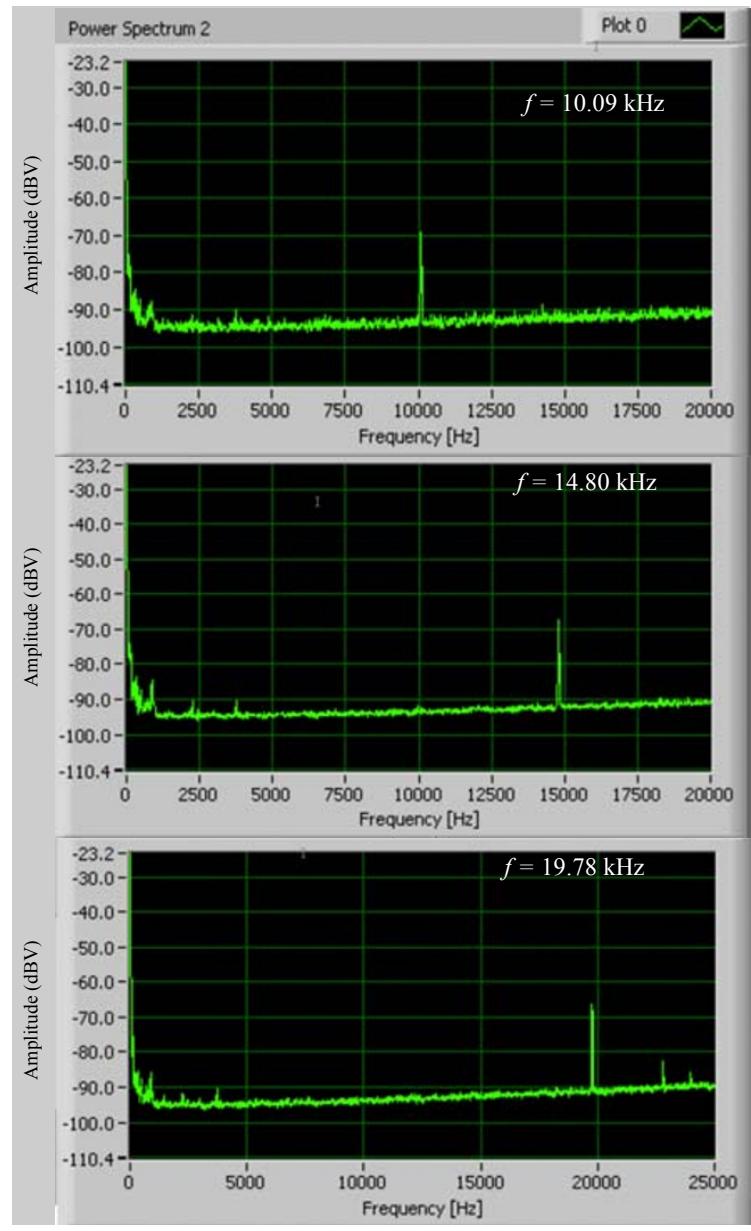

Fig. 10 Acoustic response of DFBFL based intensity-type hydrophone. 
Employing system configuration as shown in Fig. 9, we have also tested several intensity-type fiber laser hydrophones. High sensitivity and high frequency response have been achieved. As examples in Fig. 10, we show the acoustic response of a DFBFL based intensity-type hydrophone at high frequencies between $10 \mathrm{kHz}$ and $20 \mathrm{kHz}$.

\section{Conclusions}

We have presented our recent work on fiber laser based hydrophone. Implementing the interferometric-type system, homodyne digital demodulation scheme with phase generated carrier, and improved packaging schemes, we have achieved pressure sensitivity of $58.0 \mathrm{~dB} \cdot \mathrm{re} \cdot \mu \mathrm{Pa} \cdot \mathrm{Hz}^{-0.5}$ at $1 \mathrm{kHz}$ or minimum detectable acoustic pressure below $800 \mu \mathrm{Pa}$ during field test. With the intensity-type fiber laser hydrophone system, we have obtained high frequency response with benefits of reduced components, relaxed hardware requirements, simplicity, and increased robustness. This shows that the intensity-type fiber laser could be suitable to low-cost engineering and industrial applications that are not critical. Software based demodulation also contributes in term of development flexibility which is not offered by the traditional analog circuit. Due to the advantages and high performance, fiber laser hydrophones can be applied to great potential areas where utilization of ceramic hydrophones leads to cumbersomeness and impracticality. Since the current system has met general requirements, the focus is now shifted towards arrayed sensors which are more practical in real-world cases.

Open Access This article is distributed under the terms of the Creative Commons Attribution License which permits any use, distribution, and reproduction in any medium, provided the original author(s) and source are credited.

\section{References}

[1] G. A. Cranch, P. J. Nash, and C. K. Kirkendall, "Large-scale remotely interrogated arrays of fiber-optic interferometric sensors for underwater acoustic applications," IEEE Sens. J., vol. 3, no. 1, pp. 19-30, 2003.

[2] F. Kullander and C. Vahlberg, "Towards a thin and lightweight fiber optic towed array sonar," Arch. Acoust., vol. 30, no. 4, pp. 91-94, 2005.

[3] P. J. Nash, G. A. Cranch, and D. J. Hill, "Large scale multiplexed fiber-optic arrays for geophysical applications," in Proc. of SPIE, vol. 4202, pp. 55-65, 2000.

[4] A. C. L. Wong, P. A. Childs, and G. D. Peng, "Spectrally-overlapped chirped fiber Bragg grating sensor system for simultaneous two-parameter sensing," Meas. Sci. Technol., vol. 18, no. 12, pp. 3825-3832, 2007.

[5] D. Tosi, M. Olivero, and G. Perrone, "Optical microphone with fiber Bragg grating and signal processing techniques," in Proc. of SPIE, vol. 7098, pp. 70981E.1-70981E.11, 2008.

[6] H. Jun, F. Li, H. Xiao, and Y. Liu, "Fiber Bragg grating sensor array system based on digital phase generated carrier demodulation and reference compensation method," in Proc. of 1st Asia-Pacific Opt. Fiber Sensors Conf., Beijing, China, Nov. 7-9, pp. 1-4, 2008.

[7] J. H. Cole, C. Sunderman, A. B. Tveten, C. Kirkendall, and A. Dandridge, "Preliminary investigation of air-included polymer coatings for enhanced sensitivity of fiber-optic acoustic sensors," in Proc. 15th Optical Fiber Sensors Tech. Digest, Portland, USA, vol. 1, pp. 317-320, 2002.

[8] C. C. Wang, D. D. Anthony, B. T. Alan, and A. M. Yurek, "Very high responsivity fiber optic hydrophones for commercial applications," in Proc. of SPIE, vol. 2360, pp. 360-363, 1994.

[9] S. Goodman, A. Tikhomirov, and S. Foster, "Pressure compensated distributed feedback fiber laser hydrophone," in Proc. of SPIE, vol. 7004, pp. 700426, 2008.

[10] Z. Wentao, L. Yuliang, L. Fang, and X. Hao, "Fiber laser hydrophone based on double diaphragms: theory and experiment," IEEE J. Lightwave Technol., vol. 26, no. 10, pp. 1349-1352, 2008.

[11] A. I. Azmi, D. Sen, and G. D. Peng, "Sensitivity enhancement in composite cavity fiber laser hydrophone," IEEE J. Lightwave Technol., vol. 28, no. 12 , pp. 1844-1850, 2010.

[12] W. H. Loh and R. I. Laming, " $1.55 \mu \mathrm{m}$ phase-shifted distributed feedback fiber laser," Electron. Lett., vol. 31, no. 17, pp. 1440-1442, 1995.

[13] E. Ronnekleiv, "Frequency and intensity noise of single frequency fiber Bragg grating lasers," Opt. Fiber Technol., vol. 7, no. 3, pp. 206-235, 2001.

[14] G. A. Cranch and P. J. Nash, "Optical fiber hydrophones," in Lasers and Their Applications, 1 st ed., vol. 3, C. E. Webb and J. D. C. Jones, Ed. Bristol, U.K.: IOP, 2003, pp. 1839-1880. 
[15] P. Varming, V. C. Lauridsen, J. H. Povlsen, J. B. Jensen, and M. Kristensen, "Design and fabrication of Bragg grating based DFB fiber lasers operating above $1610 \mathrm{~nm}$," in Proc. Optical Fiber Communication Conference, Baltimore, USA, vol. 3, pp. 17-19, 2000.

[16] S. Ogita, Y. Kotaki, K. Kihara, M. Matsuda, H. Ishikawa, and H. Imai, "Dependence of spectral linewidth on cavity length and coupling coefficient in DFB laser," Electron. Lett., vol. 24, no. 10, pp. 613-614, 1988.

[17] E. Rønnekleiv, O. Hadeler, and G. Vienne, "Stability of an Er-Yb-doped fiber distributed-feedback laser with external reflections," Opt. Lett., vol. 24, no. 9, pp. 617-619, 1999.

[18] D. Yu. Stepanov, J. Canning, L. Poladian, R. Wyatt, G. Maxwell, R. Smith, and R. Kashyap, "Apodized distributed-feedback fiber laser," Opt. Fiber Technol., vol. 5, no. 2, pp. 209-214, 1999.

[19] A. I. Azmi, D. Sen, and G. D. Peng, "Output power and threshold gain of apodized DFB fiber laser," in Proc. of SPIE, vol. 7386, pp. 73860K.1-73860K.11, 2009.

[20] A. I. Azmi and G. D. Peng, "Performance analysis of apodized DFB fiber laser," in Proc. of Photonics Global Conf., Singapore, Dec. 8-11, pp. 1-4, 2008.

[21] Y. Dai, X. Chen, J. Sun, Y. Yao, and S. Xie, "Dual-wavelength DFB fiber laser based on a chirped structure and the equivalent phase shift method," IEEE Photonics Technol. Lett., vol. 18, no. 18, pp. 1964-1966, 2006.

[22] X. Liu, "A novel dual-wavelength DFB fiber laser based on symmetrical FBG structure," IEEE Photonics Technol. Lett., vol. 19, no. 9, pp. 632-634, 2007.

[23] A. Tikhomirov and S. Foster, "DFB FL sensor cross-coupling reduction," IEEE J. Lightwave Technol., vol. 25, no. 2, pp. 533-538, 2007.

[24] H. Kogelnik and C. V. Shank, "Coupled-wave theory of distributed feedback lasers," J. Appl. Phys., vol. 43, no. 5, pp. 2327-2335, 1972.

[25] A. Yariv, Optical Electronics in Modern Communications, 5th ed., New York: Oxford University Press Inc., 1997, pp. 619-626.

[26] N. Schunk and K. Petermann, "Numerical analysis of the feedback regimes for a single-mode semiconductor laser with external feedback," IEEE J. Quantum Electron., vol. 24, no. 7, pp. 1242-1247, 1988.

[27] S. Saito and Y. Yamamoto, "Direct observation of Lorentzian lineshape of semiconductor laser and linewidth reduction with external grating feedback," Electron. Lett., vol. 17, no. 9, pp. 325-327, 1981.

[28] L. Goldberg, A. Dandridge, R. O. Miles, T. G. Giallorenzi, and J. F. Weller, "Noise characteristics in line-narrowed semiconductor lasers with optical feedback," Electron. Lett., vol. 17, no. 19, pp. 677-678, 1981.

[29] R. Wyatt and W. J. Devlin, "10 kHz linewidth $1.5 \mu \mathrm{m}$ InGaAsP external cavity laser with $55 \mathrm{~nm}$ tuning range," Electron. Lett., vol. 19, no. 3, pp. 110-112, 1983.

[30] D. Mehuys, M. Mittelstein, and A. Yariv, "Optimised Fabry-Perot (AlGa)As quantum-well lasers tunable over $105 \mathrm{~nm}$," Electron. Lett., vol. 25, no. 2, pp. 143-145, 1989.

[31] A. Olsson and C. Tang, "Coherent optical interference effects in external-cavity semiconductor lasers," IEEE J. Quantum Electron., vol. 17, no. 8, pp. 1320-1323, 1981.

[32] J. Osmundsen and N. Gade, "Influence of optical feedback on laser frequency spectrum and threshold conditions," IEEE J. Quantum Electron., vol. 19, no. 3, pp. 465-469, 1983.

[33] G. B. Hocker, "Fiber-optic sensing of pressure and temperature," Appl. Opt., vol. 18, no. 9, pp. 1445-1448, 1979.

[34] S. Tanaka, H. Yokosuka, and N. Takahashi, "Temperature-stabilized fiber Bragg grating underwater acoustic sensor array using incoherent light," in Proc. of SPIE, vol. 5855, pp. 699-702, 2005.

[35] T. Sakoda and Y. Sonoda, "Measurement of low-frequency ultrasonic wave in water using an acoustic fiber sensor," IEEE T Ultrason. Ferr., vol. 53, no. 4, pp 761-766, 2006.

[36] B. O. Guan, Y. N. Tan, and H. Y. Tam, "Dual polarization fiber grating laser hydrophone," Opt. Express, vol. 17. no. 22, pp. 19544-19550, 2009.

[37] J. A. Bucaro, H. D. Dardy, and E. F. Carome, "Fiber-optic hydrophone," J. Acoust. Soc. Am., vol. vol. 62, no. 5, pp. 1302-1304, 1977.

[38] D. A. Jackson, R. Priest, A. Dandridge, and A. B. Tveten, "Elimination of drift in a single-node optical fiber interferometer using a piezoelectrically stretched coiled fiber," Appl. Optics, vol. 19, no. 17, pp. 2926-2929, 1980.

[39] A. Dandridge, A. Tveten, and T. Giallorenzi, "Homodyne demodulation scheme for fiber optic sensors using phase generated carrier," IEEE J. Quantum Electron., vol. 18, no. 10, pp. 1647-1653, 1982. 
[40] J. Bush, A. Cekorich, and C. K. Kirkendall, "Multichannel interferometric demodulator," in Proc. of SPIE, vol. 3180, pp. 19-29, 1997.

[41] M. Milnes, A. Tikhomirov, S. Foster, and S. Goodman, "Fast four step digital demodulation for multiplexed fiber laser sensors," in Proc. of SPIE, vol. 7004, pp. 700422.1-700422.5, 2008.

[42] D. A. Jackson, A. D. Kersey, M. Corke, and J. D. C. Jones, "Pseudoheterodyne detection scheme for optical interferometers," Electron. Lett., vol. 18, no. 25, pp. 1081-1083, 1982.

[43] J. Cole, B. Danver, and J. Bucaro, "Syntheticheterodyne interferometric demodulation," IEEE J. Quantum Electron., vol. 18, no. 4, pp. 694-697, 1982.

[44] S. C. Huang and H. Lin, "Modified phase-generated carrier demodulation compensated for the propagation delay of the fiber," Appl. Opt., vol. 46, no. 3, pp. 7594-7603, 2007.

[45] Q. Shi, et al., "The stability and consistency analysis of optical seismometer system using phase generated carrier in field application," in Proc. of SPIE, vol. 7508, pp. 75081M.1-75081M.9, 2009.

[46] K. P. Koo, A. B. Tveten, and A. Dandridge, "Passive stabilization scheme for fiber interferometers using $3 \times 3$ fiber directional couplers," Appl. Phys. Lett., vol. 41, no. 7, pp. 616-618, 1982.

[47] D. A. Brown, C. B. Cameron, R. M. Keolian, D. L. Gardner, and S. L. Garrett, "A symmetric $3 \times 3$ coupler based demodulator for fiber optic interferometric sensors," in Proc. of SPIE, vol. 1584, pp. 328-335, 1991.

[48] D. J. Hill, B. Hodder, J. De Freitas, S. D. Thomas, and L. Hickey, "DFB fiber-laser sensor developments," in Proc. of SPIE, vol. 5855, pp. 904-907, 2005.

[49] J. H. Cole, C. Kirkendall, A. Dandridge, G. Cogdell, and T. G. Giallorenzi, "Twenty-five years of interferometric fiber optics acoustic sensors at the Naval Research Laboratory," Washington Academic Science Journal, vol. 90, no. 3, pp. 40-57, 2004. 